\title{
Early Experience of Atezolizumab Plus Bevacizumab Therapy in Japanese Patients With Unresectable Hepatocellular Carcinoma in Real- world Practice
}

Yuka Hayakawa ( $\nabla$ y.hayakawa@musashino.jrc.or.jp )

Musashino Red Cross Hospital

Kaoru Tsuchiya

Musashino Red Cross Hospital: Musashino Sekijuji Byoin

Masayuki Kurosaki

Musashino Red Cross Hospital: Musashino Sekijuji Byoin

Yutaka Yasui

Musashino Red Cross Hospital: Musashino Sekijuji Byoin

Shun Kaneko

Musashino Red Cross Hospital: Musashino Sekijuji Byoin

\section{Yuki Tanaka}

Musashino Red Cross Hospital: Musashino Sekijuji Byoin

\section{Shun Ishido}

Musashino Red Cross Hospital: Musashino Sekijuji Byoin

\section{Kento Inada}

Musashino Red Cross Hospital: Musashino Sekijuji Byoin

\section{Sakura Kirino}

Musashino Red Cross Hospital: Musashino Sekijuji Byoin

Koji Yamashita

Musashino Red Cross Hospital: Musashino Sekijuji Byoin

Tsubasa Nobusawa

Musashino Red Cross Hospital: Musashino Sekijuji Byoin Hiroaki Matsumoto

Musashino Red Cross Hospital: Musashino Sekijuji Byoin

Tatsuya Kakegawa

Musashino Red Cross Hospital: Musashino Sekijuji Byoin Mayu Higuchi

Musashino Red Cross Hospital: Musashino Sekijuji Byoin

\section{Kenta Takaura}

Musashino Red Cross Hospital: Musashino Sekijuji Byoin 


\section{Shohei Tanaka}

Musashino Red Cross Hospital: Musashino Sekijuji Byoin

\section{Chiaki Maeyashiki}

Musashino Red Cross Hospital: Musashino Sekijuji Byoin

\section{Nobuharu Tamaki}

Musashino Red Cross Hospital: Musashino Sekijuji Byoin

\section{Hiroyuki Nakanishi}

Musashino Red Cross Hospital: Musashino Sekijuji Byoin

\section{Jun Itakura}

Musashino Red Cross Hospital: Musashino Sekijuji Byoin

\section{Yuka Takahashi}

Musashino Red Cross Hospital: Musashino Sekijuji Byoin

\section{Yasuhiro Asahina}

Tokyo Medical and Dental University: Tokyo Ika Shika Daigaku

\section{Ryuichi Okamoto}

Musashino Red Cross Hospital: Musashino Sekijuji Byoin

\section{Namiki Izumi}

Musashino Red Cross Hospital: Musashino Sekijuji Byoin

\section{Research Article}

Keywords: hepatocellular carcinoma, atezolizumab, bevacizumab, later-line, alpha-fetoprotein

Posted Date: August 17th, 2021

DOl: https://doi.org/10.21203/rs.3.rs-794293/v1

License: (c) (1) This work is licensed under a Creative Commons Attribution 4.0 International License. Read Full License

Version of Record: A version of this preprint was published at Investigational New Drugs on September 29th, 2021. See the published version at https://doi.org/10.1007/s10637-021-01185-4. 


\section{Abstract}

Background: We aimed to investigate the efficacy and safety of atezolizumab plus bevacizumab therapy in patients with unresectable hepatocellular carcinoma (u-HCC) based on whether they had previously received systemic therapy, as well as the association of atezolizumab plus bevacizumab with early alphafetoprotein (AFP) response in real-world practice.

Methods: A total of 52 patients with u-HCC were treated with atezolizumab plus bevacizumab between October 2020 and April 2021. The Response Evaluation Criteria in Solid Tumors (RECIST) and modified RECIST were used to evaluate radiological responses.

Results: The patients received atezolizumab plus bevacizumab as 1 st-line $(n=23), 2$ nd-line $(n=16), 3$ rdline $(n=6)$, 4th-line $(n=3)$, 5th-line $(n=3)$, or 6th-line $(n=1)$ therapy. The objective response rate and disease control rate in all patients were $18.4 \%$ and $63.2 \%$, respectively. Sixteen patients experienced no adverse events (AEs), whereas 4 patients discontinued therapy due to AEs. The median time to progression (TTP) was significantly longer among patients receiving atezolizumab plus bevacizumab as 1 st-line therapy than in patients receiving atezolizumab plus bevacizumab as later-line therapy $(P=0.02)$. Patients with an AFP response (reduction $\geq 20 \%$ from baseline) at 6 weeks had a significantly longer TTP than those without an AFP response $(P=0.02)$.

Conclusion: Patients who received atezolizumab plus bevacizumab as 1 st-line therapy had better clinical outcome than those who received atezolizumab plus bevacizumab in later lines. The AFP response at 6 weeks could be a predictor of disease progression.

\section{Introduction}

Hepatocellular carcinoma (HCC) is the most common primary liver cancer and the fourth most common cause of cancer-related deaths worldwide [1]. HCC is hypervascular, and such angiogenesis is driven by growth factors, such as vascular endothelial growth factor (VEGF). Tyrosine kinase inhibitors targeting VEGF signaling, such as sorafenib [2, 3], regorafenib [4], and lenvatinib [5], have already been approved for the treatment of unresectable HCC (u-HCC) in many countries. Cabozantinib [6] and ramucirumab [7, 8] have been approved as 1 st-line agents for u-HCC in Japan. Ramucirumab was approved for patients with a high alpha-fetoprotein (AFP) level ( $\geq 400 \mathrm{ng} / \mathrm{mL}$ ) in July 2019.

VEGF is also associated with cancer immune evasion. Based on this rationale, the combination of programmed cell death-ligand 1 (PD-L1) (atezolizumab) and VEGF (bevacizumab) pathway inhibition is believed to be the most effective molecular targeted therapy for advanced HCC. In September 2020, atezolizumab plus bevacizumab [9] was approved for patients with u-HCC in Japan and became the recommended 1st-line therapy. In Japan, agents approved for 1st- and 2nd-line therapies can be used for later-line treatment. In this study, we aimed to investigate the efficacy and safety of atezolizumab plus bevacizumab as 1st-line and later-line therapies in real-world practice. Moreover, we analyzed the factors 
associated with time to progression (TTP) and the association of atezolizumab plus bevacizumab therapy with the timing of the AFP response and disease progression.

\section{Materials And Methods Patients}

A total of 52 patients with u-HCC received atezolizumab plus bevacizumab therapy at the Musashino Red Cross Hospital between October 2020 and April 2021. Atezolizumab plus bevacizumab therapy was administered after discussions by experts at a tumor board in the institution. HCC diagnosis and evaluation of disease progression were based on guidelines proposed by the Japan Society of Hepatology [10], the American Association for the Study of Liver Disease [11], and the European Association for the Study of the Liver [12]. Written informed consent was obtained from all patients, and the ethics committee of the Musashino Red Cross Hospital approved the study in accordance with the Declaration of Helsinki.

\section{Treatment protocol}

Patients received intravenous atezolizumab $(1200 \mathrm{mg})$ plus bevacizumab $(15 \mathrm{mg} / \mathrm{kg})$ every 3 weeks until disease progression or unacceptable toxicity. Treatment interruption was performed based on the manufacturer's guidelines. Dynamic computed tomography was performed at baseline, $6-8$ weeks after atezolizumab plus bevacizumab administration, and every 6-8 weeks thereafter. Treatment response was reported based on the Response Evaluation Criteria in Solid Tumors (RECIST) [13] and the modified RECIST [14]. The AFP level was also evaluated at baseline and every 3 weeks thereafter. Adverse events (AEs) were evaluated according to the Common Terminology Criteria for Adverse Events version 5.0. To assess changes in liver function, the albumin-bilirubin (ALBI) score $[15,16]$ was analyzed at baseline and at 3,6 , and 9 weeks after treatment.

\section{Statistical analysis}

Overall survival (OS) was measured from the date of atezolizumab plus bevacizumab administration to the date of death from any cause. Patients who were lost to follow-up were censored at their last visit or contact. Patients who were still alive on April 30, 2021 were censored. Progression-free survival (PFS) was measured from the date of atezolizumab plus bevacizumab administration to the date of radiological tumor progression or death from any cause. Data are expressed as median (range) or $\mathrm{n}(\%)$. Fisher's exact test, Mann-Whitney U test, paired t-test, Kaplan-Meier method, and log-rank test were used for statistical analyses. Statistical significance was set at $P<0.05$. Statistical analyses were performed using EZR (Saitama Medical Centre, Jichi Medical University, Shimotsuke, Japan), a graphical user interface for R (The R Foundation for Statistical Computing, Vienna, Austria) [17]. 


\section{Results}

\section{Baseline patient characteristics}

The baseline characteristics of the 52 patients are presented in Table 1. Atezolizumab and bevacizumab were administered as 1st-line, 2nd-line, 3rd-line, 4th-line, 5th-line, and 6th-line treatments in 23 (44.2\%), 16 (30.8\%), 6 (11.5\%), 3 (5.8\%), 3 (5.8\%), and 1 (1.9\%) patient, respectively. Among the 16 patients who received atezolizumab plus bevacizumab as 2 nd-line treatment, 15 were treated with lenvatinib as 1 st-line treatment and 1 patient who had been treated with cytotoxic agents as systemic chemotherapy received ramucirumab as the 1 st-line molecular targeted agent. Among the 6 patients who received atezolizumab plus bevacizumab as 3rd-line treatment, 3 were previously treated with lenvatinib and sorafenib, 2 received sorafenib and regorafenib, and 1 received axitinib + avelumab (NCT03289533) and lenvatinib. Among the 3 patients who received atezolizumab plus bevacizumab as 4th-line treatment, 2 were treated with sorafenib, regorafenib, and lenvatinib and 1 received lenvatinib, sorafenib, and regorafenib. Among the 3 patients who received atezolizumab plus bevacizumab as 5th-line treatment, 1 was treated with sorafenib, regorafenib, lenvatinib, and ramucirumab, 1 was treated with sorafenib, cabozantinib, lenvatinib, and ramucirumab, and 1 received lenvatinib, sorafenib, ramucirumab, and regorafenib. The 1 patient who received atezolizumab plus bevacizumab as 6th-line treatment was previously treated with axitinib plus avelumab (NCT03289533), cabozantinib, lenvatinib, ramucirumab, and sorafenib. 
Table 1

Baseline characteristics

\begin{tabular}{|c|c|}
\hline & $\mathrm{n}=52$ \\
\hline Age (years), median (range) & $73(24-89)$ \\
\hline Sex, male/female & $42(80.8) / 10(19.2)$ \\
\hline Etiology, HBV/HCV/alcohol/others & $10(19.2) / 20(38.5) / 13(25.0) / 9(17.3)$ \\
\hline ALBI score, median (range) & $-2.24(-3.28$ to -1.37$)$ \\
\hline Modified ALBI grade, $1 / 2 a / 2 b / 3$ & $12(23.1) / 13(25.0) / 26(50.0) / 1$ (1.9) \\
\hline Child-Pugh class, A/B & $48(92.3) / 4(7.7)$ \\
\hline ECOG-PS, 0/1 & $30(57.7) / 22(42.3)$ \\
\hline BCLC stage, A/B/C & $0(0) / 29(55.8) / 23(44.2)$ \\
\hline Major portal invasion, yes/no & $6(11.5) / 46(88.5)$ \\
\hline Extrahepatic spread, yes/no & $17(32.7) / 35(67.3)$ \\
\hline $\begin{array}{l}\text { Baseline AFP concentration }(\mathrm{ng} / \mathrm{mL}) \text {, } \\
\text { median (range) }\end{array}$ & $192.7(1.6-79739.1)$ \\
\hline $\begin{array}{l}\text { Clinical course, } 1 \text { st-line/2nd-line/3rd-line/4th-line/5th- } \\
\text { line/6th-line }\end{array}$ & $\begin{array}{l}23(44.2) / 16(30.8) / 6(11.5) / 3(5.8) / 3 \\
(5.8) / 1(1.9)\end{array}$ \\
\hline \multicolumn{2}{|l|}{ Data are presented as $\mathrm{n}(\%)$ unless otherwise indicated. } \\
\hline \multicolumn{2}{|c|}{$\begin{array}{l}\text { Abbreviations: HBV, hepatitis B virus; HCV, hepatitis C virus; ALBI score, albumin-bilirubin score; ECOG- } \\
\text { PS, Eastern Cooperative Oncology Group-Performance status; BCLC, Barcelona Clinic Liver Cancer; } \\
\text { AFP, alpha-fetoprotein. }\end{array}$} \\
\hline
\end{tabular}

Therapeutic efficacy

At the end of the data cutoff (April 30, 2021), the median duration of follow-up was 126 days (range, 2187 days). The median number of courses of atezolizumab plus bevacizumab was 4 (range, 1-10). During the observation period, 28 patients continued atezolizumab plus bevacizumab therapy and 24 patients discontinued atezolizumab plus bevacizumab therapy because of progressive disease (PD) ( $n=$ 18 ) or AEs $(n=6)$. Two patients died from HCC progression, and one patient died of cerebral hemorrhage. The median OS was not reached, and the median PFS was 4.0 months (Figure 1). In 23 patients who received atezolizumab plus bevacizumab as 1 st-line treatment, the median OS and PFS were not reached, and the PFS at 3 months was $78.7 \%$ (Figure 2). 
Radiological evaluation after atezolizumab plus bevacizumab administration was performed in 38 patients. With regard to the best antitumor response according to the RECIST, 1 patient achieved complete response (CR), 6 patients achieved partial response (PR), 17 patients had stable disease (SD), and 14 patients had PD. The objective response rate (ORR) and disease control rate (DCR) were $18.4 \%$ and $63.2 \%$, respectively. With regard to the best antitumor response according to the modified RECIST, 1 patient achieved CR, 9 patients achieved PR, 15 patients had SD, and 13 patients had PD. The ORR and DCR were $26.3 \%$ and $65.8 \%$, respectively.

Treatment after atezolizumab plus bevacizumab

During the observation period, 22 of 24 patients (91.7\%) who discontinued atezolizumab plus bevacizumab received further anticancer therapy (Table 2). The other 2 patients (8.3\%) received palliative care. The transition rates to post-treatment among patients receiving atezolizumab plus bevacizumab as 1 st-line therapy $(n=4)$ and those receiving atezolizumab plus bevacizumab as later-line therapy $(n=20)$ were $100 \%$ and $90 \%$, respectively.

Table 2

Treatment after discontinuation of atezolizumab plus bevacizumab $(n=22)$

\begin{tabular}{|l|l|}
\hline & $\mathbf{n}(\%)$ \\
\hline Lenvatinib & $6(27.4)$ \\
\hline Cabozantinib & $5(22.7)$ \\
\hline Sorafenib & $5(22.7)$ \\
\hline Regorafenib & $1(4.5)$ \\
\hline Ramucirumab & $2(9.1)$ \\
\hline Hepatic arterial infusion chemotherapy & $2(9.1)$ \\
\hline Transarterial infusion chemotherapy & $1(4.5)$ \\
\hline
\end{tabular}

Changes in liver function during atezolizumab plus bevacizumab therapy

The changes in the ALBI score in 28 patients who received atezolizumab plus bevacizumab for more than 9 weeks are shown in Fig. 3. The median ALBI scores at baseline, 3 weeks, 6 weeks, and 9 weeks were 2.16 (range, -3.28 to -1.59 ), -2.13 (range, -3.12 to -1.17 ), -2.16 (range, -3.07 to -1.13 ), and -1.98 (range, -3.19 to -1.26 ), respectively. There were no significant differences in the median ALBI scores at baseline and 3 weeks $(P=0.26)$, at baseline and 6 weeks $(P=0.72)$, or at baseline and 9 weeks $(P=0.11)$. Among patients receiving atezolizumab plus bevacizumab as 1 st-line treatment $(n=14)$, the median ALBI scores at baseline, 3 weeks, 6 weeks, and 9 weeks were -2.37 (range, -3.28 to -1.59 ), -2.35 (range, 3.12 to -1.17 ), -2.40 (range, -3.07 to -1.13 ), and -2.40 (range, -3.19 to -1.26 ), respectively. In 
patients receiving atezolizumab plus bevacizumab as $2 n d$ - or later-line therapy $(n=14)$, the median ALBI scores at baseline, 3 weeks, 6 weeks, and 9 weeks were -2.13 (range, -2.44 to -1.71 ), -2.10 (range, 3.03 to -1.66 ), -2.07 (range, -2.73 to -1.60 ), and -1.92 (range, -3.02 to -1.26 ), respectively. There were no significant differences in the median ALBI scores at baseline and at 6 weeks or at baseline and at 9 weeks in either group (Fig. 4).

Adverse events during atezolizumab plus bevacizumab therapy

AEs observed during atezolizumab plus bevacizumab therapy are shown in Table 3. Any grade AE was observed in 36 of the 52 patients. The rates of total AEs in patients receiving atezolizumab plus bevacizumab as 1 st-line therapy $(n=23)$ and those receiving atezolizumab plus bevacizumab as $2 n d$ - or later-line therapy $(n=29)$ were $60.9 \%$ and $75.9 \%$, respectively $(P=0.37)$. When comparing AEs with an incidence of $10 \%$ or more, the rates of AEs were $56.5 \%$ in patients receiving atezolizumab plus bevacizumab as 1 st-line therapy and $75.9 \%$ in patients receiving atezolizumab plus bevacizumab as $2 \mathrm{nd}$ or later-line therapy $(P=0.23)$. No infusion reaction was reported in any patient. During the observation period, six patients discontinued atezolizumab plus bevacizumab therapy owing to AEs, as follows: two patients with transaminase increase, one patient with interstitial pneumonia, one patient with meningitis and transaminase increase, one patient with renal dysfunction, and one patient with gastrointestinal bleeding. Four of the six patients were treated with corticosteroids. The rates of discontinuation due to AEs among patients treated with atezolizumab plus bevacizumab as $1 \mathrm{st}$-line and 2 nd- or later-line therapy were $8.7 \%$ and $13.8 \%$, respectively $(P=0.68)$. No AEs other than those reported in the IMbrave150 trial were observed. 
Table 3

Adverse events

\begin{tabular}{|lll|}
\hline & Any grade & Grade $\geq \mathbf{3}$ \\
& $\mathbf{n}(\%)$ & $\mathbf{n}(\%)$ \\
\hline Hypertension & $12(23.1)$ & $0(0)$ \\
\hline Fatigue & $14(26.9)$ & $1(1.9)$ \\
\hline Diarrhea & $5(9.6)$ & $0(0)$ \\
\hline Decreased appetite & $8(15.4)$ & $1(1.9)$ \\
\hline Fever & $11(21.2)$ & $1(1.9)$ \\
\hline Rash & $3(5.8)$ & $0(0)$ \\
\hline Nausea & $5(9.6)$ & $1(1.9)$ \\
\hline Edema & $8(15.4)$ & $0(0)$ \\
\hline Ascites & $3(5.8)$ & $0(0)$ \\
\hline Transaminase increase & $7(13.5)$ & $3(5.8)$ \\
\hline Total bilirubin increase & $4(7.7)$ & $0(0)$ \\
\hline Proteinuria & $7(13.5)$ & $0(0)$ \\
\hline Thromboembolism & $0(0)$ & $0(0)$ \\
\hline Epistaxis, subcutaneous hemorrhage, or gastrointestinal bleeding & $8(15.4)$ & $1(1.9)$ \\
\hline Interstitial pneumonia & $1(1.9)$ & $1(1.9)$ \\
\hline Renal dysfunction & $1(1.9)$ & $1(1.9)$ \\
\hline Adrenal insufficiency & $1(1.9)$ & $0(0)$ \\
\hline Meningitis & $1(1.9)$ & $1(1.9)$ \\
\hline Infusion reaction & $0(0)$ & $0(0)$ \\
\hline
\end{tabular}

Clinical outcomes in the 1 st-line group and the 2 nd- or later-line group

To reveal the clinical outcome in patients who received atezolizumab plus bevacizumab as 2 nd- or laterline treatment, we compared patients who received atezolizumab plus bevacizumab as 1 st-line treatment (1st-line group; $n=17$ ) and those who received atezolizumab plus bevacizumab as 2 nd- or later-line treatment ( $2 n d$ - or later-line group; $n=21$ ) in terms of TTP. The baseline characteristics are shown in Table 4. There were no significant differences in age, sex, etiology, or pretreatment AFP between the two 
groups. There were no statistically significant differences in pretreatment ALBI score or ECOG-PS, but baseline liver function and ECOG-PS were slightly worse in 2nd- or later-line group. The proportions of patients receiving a full dose of atezolizumab plus bevacizumab in the 1st-line group and the 2 nd- or later-line group were $70.6 \%$ and $57.1 \%$, respectively $(P=0.51)$. Among 17 patients who received atezolizumab plus bevacizumab as 1st-line treatment, 1 patient achieved CR, 4 patients achieved PR, 8 patients had SD, and 4 patients had PD according to the RECIST. The ORR was $29.4 \%$ and the DCR was 76.5\% according to the RECIST. According to the modified RECIST, 1 patient achieved CR, 5 achieved PR, 7 had SD, and 4 had PD. The ORR was $35.3 \%$ and the DCR was $76.5 \%$ using the modified RECIST. Among the 21 patients who received atezolizumab plus bevacizumab as 2nd- or later-line treatment, 2 achieved PR, 9 had SD, and 10 had PD according to the RECIST. The ORR was $9.5 \%$ and the DCR was $52.3 \%$ using the RECIST. According to the modified RECIST, 4 patients achieved PR, 8 had SD, and 9 had PD. The ORR was $19.0 \%$ and the DCR was $57.1 \%$ using the modified RECIST. The median TTP was significantly longer in the 1 st-line group than in the 2 nd- or later-line group (not reached vs. 2.6 months, $P=0.02$ ) (Fig. 5).

We next analyzed the factors associated with TTP in patients receiving atezolizumab plus bevacizumab $(\mathrm{n}=38)$. In the univariate analysis, receiving atezolizumab plus bevacizumab as $2 \mathrm{nd}$ - or later-line therapy was the only significant factor (hazard ratio $3.82,95 \%$ confidence interval $1.11-13.17, P=0.03$ ) (Table 5). 
Table 4

Baseline characteristics in the 1st-line group and the 2nd- or later-line group

\begin{tabular}{|c|c|c|c|}
\hline & 1st-line group $(n=17)$ & $\begin{array}{l}\text { 2nd- or later-line group } \\
(n=21)\end{array}$ & $P$ \\
\hline Age (years), median (range) & $73(61-79)$ & $77(34-86)$ & 0.78 \\
\hline Sex, male (\%) & $12(70.6)$ & $18(85.7)$ & 0.43 \\
\hline $\begin{array}{l}\text { Etiology, HBV/HCV/alcohol/others } \\
(\%)\end{array}$ & $\begin{array}{l}3(17.6) / 8(47.1) / 5 \\
(29.4) / 1(5.9)\end{array}$ & $\begin{array}{l}3(14.3) / 5(23.8) / 7 \\
(33.3) / 6(28.6)\end{array}$ & 0.46 \\
\hline ECOG-PS, 0/1 (\%) & $13(76.5) / 4(23.5)$ & $9(42.9) / 12(57.1)$ & 0.05 \\
\hline BCLC stage B (\%) & $11(64.7)$ & $8(38.1)$ & 0.19 \\
\hline Major portal invasion (\%) & $3(17.6)$ & $3(14.3)$ & $\overrightarrow{0.99}$ \\
\hline Extrahepatic metastasis (\%) & $3(17.6)$ & $10(47.6)$ & 0.09 \\
\hline ALBI score, median (range) & $-2.39(-3.28$ to -1.60$)$ & $-2.14(-2.76$ to -1.57$)$ & 0.06 \\
\hline Child-Pugh A (\%) & $17(100)$ & $19(90.5)$ & 0.49 \\
\hline Alb (g/dL, median) & $3.6(2.8-4.7)$ & $3.4(2.8-4.1)$ & 0.05 \\
\hline T-Bil (mg/dL, median) & $0.7(0.4-1.2)$ & $0.6(0.3-2.6)$ & 0.52 \\
\hline PT (\%, median) & $95(72-114)$ & $94(57-120)$ & 0.99 \\
\hline AST (U/L, median) & $50(24-123)$ & $42(3-161)$ & 0.59 \\
\hline ALT (U/L, median) & $34(14-123)$ & $26(1-73)$ & 0.25 \\
\hline $\begin{array}{l}\text { eGFR }\left(\mathrm{mL} / \mathrm{min} / 1.73 \mathrm{~m}^{2}\right) \text {, median } \\
\text { (range) }\end{array}$ & $59.9(37.3-96.3)$ & $69.9(37.5-100.8)$ & 0.15 \\
\hline $\begin{array}{l}\text { Urine protein-to-creatine ratio, median } \\
\text { (range) }\end{array}$ & $0.07(0-1.53)$ & $0.09(0.03-4.14)$ & 0.12 \\
\hline AFP (ng/mL), median (range) & $247(1.6-9194.5)$ & $186(4.8-37477.2)$ & 0.35 \\
\hline $\mathrm{DCP}(\mathrm{mAU} / \mathrm{mL})$, median (range) & $144.5(21.9-313273.7)$ & 1067.5(93-200499) & 0.71 \\
\hline \multicolumn{4}{|c|}{$\begin{array}{l}\text { Abbreviations: HBV, hepatitis B virus; HCV, hepatitis C virus; ECOG-PS, Eastern Cooperative Oncology } \\
\text { Group-Performance Status; BCLC, Barcelona Clinic Liver Cancer; ALBI, albumin-bilirubin; Alb, albumin; } \\
\text { T-Bil, total bilirubin; PT, prothrombin; AST, aspartate aminotransferase; ALT, alanine aminotransferase; } \\
\text { eGFR, estimated glomerular filtration rate; AFP, alpha-fetoprotein; DCP, des-ү-carboxy prothrombin. }\end{array}$} \\
\hline \multicolumn{4}{|c|}{ 1st-line group: received atezolizumab plus bevacizumab as 1 st-line therapy } \\
\hline $\begin{array}{l}\text { 2nd- or later-line group: received atezo } \\
\text { therapy }\end{array}$ & Imab plus bevacizumab & 2nd-, 3rd-, 4th-, 5th-, or 6 t & \\
\hline
\end{tabular}


Table 5

Factors associated with time to progression in patients treated with atezolizumab plus bevacizumab $(\mathrm{n}=$ 38)

\begin{tabular}{|c|c|c|c|}
\hline & \multicolumn{3}{|c|}{ Univariate analysis } \\
\hline & $\mathrm{HR}$ & $95 \% \mathrm{Cl}$ & $P$ value \\
\hline Age $\geq 75$ years & 0.81 & $0.32-2.02$ & 0.66 \\
\hline Sex, male & 1.11 & $0.31-3.85$ & 0.86 \\
\hline Etiology, non-viral & 2.07 & $0.81-5.30$ & 0.12 \\
\hline ALBI grade $2 b$ & 0.66 & $0.26-1.64$ & 0.33 \\
\hline ECOG-PS >0 & 1.50 & $0.60-3.71$ & 0.37 \\
\hline BCLC stage C & 2.32 & $0.87-6.13$ & 0.08 \\
\hline Major portal invasion & 1.17 & $0.33-4.13$ & 0.80 \\
\hline Extrahepatic spread & 2.11 & $0.85-5.22$ & 0.10 \\
\hline $\mathrm{AFP} \geq 400 \mathrm{ng} / \mathrm{mL}$ & 1.50 & $0.61-3.72$ & 0.37 \\
\hline Atezolizumab plus bevacizumab as 2 nd- or later-line treatment & 3.82 & $1.11-13.17$ & 0.03 \\
\hline Dose reduction or discontinuation & 0.94 & $0.36-2.43$ & 0.90 \\
\hline \multicolumn{4}{|c|}{$\begin{array}{l}\text { Abbreviations: HR, hazard ratio; CI, confidence interval; ECOG-PS, Eastern Cooperative Oncology } \\
\text { Group-Performance Status; BCLC, Barcelona Clinic Liver Cancer; ALBI, albumin-bilirubin score; AFP, } \\
\text { alpha-fetoprotein. }\end{array}$} \\
\hline
\end{tabular}

The association between the AFP response and therapeutic efficacy in patients receiving atezolizumab plus bevacizumab therapy

We investigated the AFP response as a predictor of DCR according to RECIST in patients receiving atezolizumab plus bevacizumab therapy. The AFP response was defined as a reduction in AFP from the baseline of $\geq 20 \%$. Thirty-two patients continued atezolizumab plus bevacizumab therapy for more than 6 weeks and were included in the analysis. At 3 weeks, 12 patients showed an AFP response. The AFP response at 3 weeks had a positive predictive value (PPV) of $91.7 \%$ and a negative predictive value (NPV) of $40.0 \%$. At 6 weeks, 12 patients showed an AFP response. The AFP response at 6 weeks had a PPV of $91.7 \%$ and an NPV of $40.0 \%$. The TTPs of patients with and without an AFP response at 3 weeks and 6 weeks are presented in Fig. 6 . There were no significant differences in the median TTP between patients with and without an AFP response at 3 weeks ( 3.8 months vs. 4.8 months, $P=0.09$ ). By contrast, there was a significant difference in the median TTP between patients with and without an AFP response at 6 weeks ( 2.6 months vs. 4.7 months, $P=0.02$ ). The results were similar when patients with a baseline AFP $<20 \mathrm{ng} / \mathrm{mL}$ were excluded. Although there were no significant differences in the median TTP between 
patients with and without an AFP response at 3 weeks ( 3.8 months vs. 4.1 months, $P=0.08)$, there was a significant difference in the median TTP between patients with and without an AFP response at 6 weeks (2.5 months vs. 4.7 months, $P=0.001$ ) (Fig. 7).

We also analyzed the association between the AFP response at 6 weeks and therapeutic efficacy stratified by patients with baseline AFPs $<20 \mathrm{ng} / \mathrm{mL}, 20-400 \mathrm{ng} / \mathrm{mL}$, and $>400 \mathrm{ng} / \mathrm{mL}$ (Table 6). Among 10 patients with a baseline AFP $<20 \mathrm{ng} / \mathrm{mL}, 3$ had an AFP response at 6 weeks. According to the RECIST, 2 patients achieved PR and 1 had SD. Among 10 patients with a baseline AFP $20-400 \mathrm{ng} / \mathrm{mL}, 4$ had an AFP response at 6 weeks. One patient achieved PR, 2 had SD, and 1 had PD. Among 12 patients with a baseline AFP $>400 \mathrm{ng} / \mathrm{mL}, 5$ had an AFP response at 6 weeks. One patient achieved CR, 1 achieved PR, and 3 patients had SD. There were no significant differences in the median TTP among patients with baseline AFPs $<20 \mathrm{ng} / \mathrm{mL}, 20-400 \mathrm{ng} / \mathrm{mL}$, and $>400 \mathrm{ng} / \mathrm{mL}$ (5.2 months, 4.0 months, and 4.8 months, respectively; $\mathrm{P}=0.19$ ) in patients with an AFP response at 6 weeks (Fig. 8).

Table 6

Positive predictive value and negative predictive value of the AFP response at 6 weeks classified by baseline AFP

\begin{tabular}{|lll|}
\hline Baseline AFP & Positive predictive value (PPV) & Negative predictive value (NPV) \\
\hline$<20 \mathrm{ng} / \mathrm{mL}(\mathrm{n}=10)$ & $100 \%$ & $28.6 \%$ \\
\hline $20-400 \mathrm{ng} / \mathrm{mL}(\mathrm{n}=10)$ & $75 \%$ & $50 \%$ \\
\hline$>400 \mathrm{ng} / \mathrm{mL}(\mathrm{n}=12)$ & $100 \%$ & $42.9 \%$ \\
\hline Abbreviation: AFP, alpha-fetoprotein.
\end{tabular}

\section{Discussion}

Atezolizumab plus bevacizumab is recommended as a 1st-line therapy for advanced HCC in many guidelines, including the ASCO [18] and ESMO guidelines [19]. Some recent studies have reported the efficacy and safety of atezolizumab plus bevacizumab in Japanese patients with u-HCC in real-world practice [20-22]. To the best of our knowledge, this is the first report to reveal the difference in clinical outcome between patients receiving atezolizumab plus bevacizumab as 1 st-line and 2 nd- or later-line therapies and the association between the timing of the AFP response and therapeutic efficacy.

We included patients who received atezolizumab plus bevacizumab as 2 nd- or later-line therapy in this study. The IMbrave150 trial [23] included only patients who had not previously received systemic therapy, and the ORR and DCR were $27.3 \%$ and $73.6 \%$, respectively, according to the RECIST. Kuzuya et al. [20] reported an ORR of $12.5 \%$ and a DCR of $75.0 \%$ according to the RECIST in patients treated with atezolizumab plus bevacizumab after lenvatinib failure. Hiraoka et al. [21] reported that the ORRs and DCRs were $12.2 \%$ and $82.9 \%$, respectively, in patients who received atezolizumab plus bevacizumab as 1st-line therapy, and $9.7 \%$ and $77.8 \%$, respectively, in patients who received atezolizumab plus bevacizumab as 2 nd- or later-line therapy, according to the RECIST. Iwamoto et al. [22] reported that the ORRs and DCRs were $36.8 \%$ and $94.5 \%$ in molecular targeted agent (MTA)-naïve patients, respectively, 
and $34.3 \%$ and $71.9 \%$ in patients treated with MTAs, respectively, using the modified RECIST. These reports showed that there were no significant differences in the clinical response to atezolizumab plus bevacizumab in patients with and without a past history of systemic treatment. In our study, the median TTP was significantly shorter in the 2nd- or later-line group than in the first-line group. In patients previously treated with other agents, dose modification and interruption of bevacizumab were more frequent than in patients receiving atezolizumab plus bevacizumab as 1st-line therapy. The slight difference in baseline ALBI scores between two groups may also have an impact on TTP. However, there were no significant differences in the rate of $A E s$ between the two groups.

Previous reports reported deterioration in liver function during the early period among patients treated with sorafenib [24] and lenvatinib [25]. Sangro et al. [26] reported that patients receiving nivolumab were more likely to have preserved liver function than patients receiving sorafenib. Kudo et al. [27] reported that ramucirumab did not worsen the ALBI score during the early treatment period. Changes in liver function during atezolizumab plus bevacizumab therapy were not examined in the IMbrave150 trial. Kuzuya et al. [20] reported that the ALBI score of patients receiving atezolizumab plus bevacizumab at 2 weeks was worse than that at baseline but improved at 3 and 6 weeks without any special treatment. Hiraoka et al. [21] also reported that the ALBI score was significantly worsened at 3 weeks after the introduction of atezolizumab plus bevacizumab but recovered at 6 weeks. In our study, the ALBI score at 3 weeks transiently worsened compared to baseline in patients receiving atezolizumab plus bevacizumab as 1 stline treatment but improved at 6 weeks and 9 weeks. The temporary worsening of liver function may be related to the therapeutic response, which leads to appetite loss and fatigue during the early treatment period. All patients $(n=11)$ with deterioration of the ALBI score at 3 weeks in the 1 st-line group had PR or SD. Liver function and these AEs improved spontaneously at 6 and 9 weeks. Atezolizumab plus bevacizumab therapy can be maintained while preserving liver function in many patients.

In this study, we focused on the AFP response at 3 and 6 weeks after the administration of the combination therapy. There were no significant differences in the median TTP between patients with and without an AFP response at 3 weeks. By contrast, there was a significant difference in the median TTP between patients with and without an AFP response at 6 weeks. Kuzuya et al. reported that an early AFP response is useful to predict response to sorafenib [28] and lenvatinib [29]. Kodama et al. [30] reported that patients with sustained AFP reduction at 4 weeks after lenvatinib administration demonstrated a higher objective response. Lee et al. [31] reported that an AFP reduction $>10 \%$ within 4 weeks is an independent predictor of the best objective response to immune checkpoint inhibitor therapy. However, in patients receiving atezolizumab plus bevacizumab therapy, the antitumor response is delayed in some patients, and early treatment decisions based on the AFP response should be avoided.

In Japan, five regimens (sorafenib, regorafenib, lenvatinib, ramucirumab if AFP $\geq 400 \mathrm{ng} / \mathrm{mL}$, and cabozantinib) can be administered after atezolizumab plus bevacizumab therapy based on the national health insurance guidelines. Because of the small cohort and short observation period, we could not examine the established clinical outcomes of post-combination therapies in real-world practice. Among the patients who received lenvatinib after combination therapy, the ORR and DCR were $50 \%$ and $75 \%$, 
respectively. Aoki et al. [32] revealed that lenvatinib demonstrated considerable antitumor effects with acceptable safety in patients with progressive and u-HCC when administered immediately after PD-1/PDL1 blockade failure (ORR 55.6\%, DCR 86.1\%). Further multicenter studies on treatment after atezolizumab plus bevacizumab therapy are necessary to develop an effective treatment strategy for patients with uHCC.

There are certain limitations to our study. The study was a retrospective single-center study with a small number of patients and a short observation period, and tumor response was not evaluated in all patients.

To our knowledge, our report is the first to reveal the difference in clinical outcome between 1 st-line and 2nd- or later-line atezolizumab plus bevacizumab therapy and the association between the timing of the AFP response and therapeutic efficacy. Our data can provide useful information to develop useful therapeutic strategies for patients with u-HCC and effectively administer combination therapy.

\section{Conclusion}

Atezolizumab plus bevacizumab therapy was found to have similar radiological responses to the phase III IMbrave150 trial regimen in u-HCC patients when administered as 1st-line therapy. Patients receiving atezolizumab plus bevacizumab therapy as $1 \mathrm{st}$-line therapy tended to have longer TTP than those receiving atezolizumab plus bevacizumab as 2 nd- or later-line therapy. The AFP response at 6 weeks could be a predictive indicator of disease progression.

\section{Declarations}

\section{Acknowledgement}

We thank the patients and medical staff who contributed to this study.

\section{Conflict of Interest}

Kaoru Tsuchiya, Masayuki Kurosaki, and Namiki Izumi received advisory board fees and honoraria from the speakers' bureau from Bayer, Eisai, Eli Lilly Japan, and Roche. The Japanese Ministry of Health, Labour and Welfare had no role in the design of the study; in the collection, analyses, or interpretation of data; in the writing of the manuscript; or in the decision to publish the results.

\section{Funding}

This study was supported by a grant-in-aid from the Japanese Ministry of Health, Labour and Welfare and from the Japan Agency for Medical Research and Development (JP19fk0210025h0003).

\section{Ethical approval}


The study was conducted according to the guidelines of the Declaration of Helsinki and approved by the Ethics Committee of Musashino Red Cross Hospital (No. 28077).

\section{Informed consent}

Informed consent was obtained from all subjects involved in the study.

\section{Availability of data and materials}

The data presented in this study are available upon request from the corresponding author.

\section{Code availability}

Not applicable.

\section{Author's contribution}

Study design: Yuka Hayakawa and Kaoru Tsuchiya

Data collection: Yuka Hayakawa, Kaoru Tsuchiya, Masayuki Kurosaki, Yutaka Yasui, Shun Kaneko, Yuki Tanaka, Shun Ishido, Kento Inada, Sakura Kirino, Koji Yamashita, Tsubasa Nobusawa, Hiroaki Matsumoto, Tatsuya Kakegawa, Mayu Higuchi, Kenta Takaura, Shohei Tanaka, Chiaki Maeyashiki, Nobuharu Tamaki, Hiroyuki Nakanishi, Jun Itakura, Yuka Takahashi and Namiki Izumi

Data analysis: Yuka Hayakawa and Kaoru Tsuchiya

Data interpretation: Yuka Hayakawa, Kaoru Tsuchiya, Masayuki Kurosaki, Shun Kaneko, Yutaka Yasui, Yasuhiro Asahina, Ryuichi Okamoto and Namiki Izumi

Manuscript writing: Yuka Hayakawa and Kaoru Tsuchiya

Manuscript revision: Masayuki Kurosaki, Shun Kaneko, Yutaka Yasui, Yasuhiro Asahina, Ryuichi Okamoto and Namiki Izumi

All authors have read and agreed to the published version of the manuscript.

\section{References}

1. Bray F, Ferlay J, Soerjomataram I, et al. Global cancer statistics 2018: GLOBOCAN estimates of incidence and mortality worldwide for 36 cancers in 185 countries. CA Cancer J Clin. 2018;68:394424.

2. Llovet JM, Ricci S, Mazzaferro V, et al. Sorafenib in advanced hepatocellular carcinoma. N Engl J Med. 2008;359:378-390. 
3. Cheng AL, Kang YK, Chen Z, et al. Efficacy and safety of sorafenib in patients in the Asia-Pacific region with advanced hepatocellular carcinoma: a phase III randomised, double-blind, placebocontrolled trial. Lancet Oncol. 2009;10:25-34.

4. Bruix J, Qin S, Merle P, et al. Regorafenib for patients with hepatocellular carcinoma who progressed on sorafenib treatment (RESORCE): a randomised, double-blind, placebo-controlled, phase 3 trial. Lancet. 2017;389:56-66.

5. Kudo M, Finn RS, Qin S, et al. Lenvatinib versus sorafenib in first-line treatment of patients with unresectable hepatocellular carcinoma: a randomised phase 3 non-inferiority trial. Lancet. 2018;391:1163-1173.

6. Abou-Alfa GK, Meyer T, Cheng AL, et al. Cabozantinib in patients with advanced and progressing hepatocellular carcinoma. N Engl J Med. 2018;379:54-63.

7. Park JO, Ryoo BY, Yen CJ, et al. Second-line ramucirumab therapy for advanced hepatocellular carcinoma (REACH): An East Asian and non-East Asian subgroup analysis. Oncotarget. 2016;7:75482-75491.

8. Zhu AX, Kang YK, Yen CJ, et al. Ramucirumab in advanced hepatocellular carcinoma and elevated alpha-fetoprotein following sorafenib (REACH-2): A randomised, double-blind, placebocontrolled phase 3 trial. Lancet Oncol. 2019;20:282-296.

9. Finn RS, Ducreux M, Qin S, et al. IMbrave150: A randomized phase III study of $1 \mathrm{~L}$ atezolizumab plus bevacizumab vs sorafenib in locally advanced or metastatic hepatocellular carcinoma. J Clin Oncol. 2018;36:TPS4141-TPS4141.

10. Kokudo N, Takemura N, Hasegawa K, et al. Clinical practice guidelines for hepatocellular carcinoma: The Japan Society of Hepatology 2017 (4th JSH-HCC guidelines) 2019 update. Hepatol Res. 2019;49:1109-1113.

11. Marrero JA, Kulik LM, Sirlin C, et al. Diagnosis, staging and management of hepatocellular carcinoma: 2018 practice guidance by the American Association for the Study of Liver Diseases. Hepatology. 2018;68:723-750.

12. European Association for the Study of the Liver. EASL Clinical Practice Guidelines: management of hepatocellular carcinoma. J Hepatol. 2018;69:182-236.

13. Eisenhauer EA, Therasse $P$, Bogaerts $J$, et al. New response evaluation criteria in solid tumours: revised RECIST guideline (version 1.1). Eur J Cancer. 2009 Jan;45:228-247.

14. Lencioni R, Llovet JM. Modified RECIST (mRECIST) assessment for hepatocellular carcinoma. Semin Liver Dis. 2010;30:52-60.

15. Johnson PJ, Berhane S, Kagebayashi C, et al. Assessment of liver function in patients with hepatocellular carcinoma: a new evidence-based approach-the ALBI grade. J Clin Oncol. 2014;33:550-558.

16. Hiraoka A, Kumada T, Tsuji K, et al. Validation of modified ALBI Grade for more detailed assessment of hepatic function in hepatocellular carcinoma patients: A multicenter analysis. Liver Cancer. 2019;8:121-129. 
17. Kanda Y. Investigation of the freely available easy-to-use software 'EZR' for medical statistics. Bone Marrow Transplant. 2013;48:452-445.

18. Gordan JD, Kennedy EB, Abou-Alfa GK, et al. Systemic Therapy for Advanced Hepatocellular Carcinoma: ASCO Guideline. J Clin Oncol. 2020;38:4317-4345.

19. Vogel A, Martinelli E. Updated treatment recommendations for hepatocellular carcinoma (HCC) from the ESMO Clinical Practice Guidelines. Ann Oncol. 2021;32:801-805.

20. Kuzuya T, Kawabe N, Hashimoto S, et al. Initial experience of atezolizumab plus bevacizumab for advanced hepatocellular carcinoma in clinical practice. Cancer Diagnosis \& Prognosis. 2021;1:1922.

21. Hiraoka A, Kumada T, Tada T, et al. Atezolizumab plus bevacizumab treatment for unresectable hepatocellular carcinoma: Early clinical experience. Cancer Rep (Hoboken). 2021;e1464.

22. Iwamoto H, Shimose $S$, Noda $Y$, et al. Initial experience of atezolizumab plus bevacizumab for unresectable hepatocellular carcinoma in real-world clinical practice. Cancers. 2021;13:2786.

23. Finn RS, Qin S, Ikeda $M$, et al. Atezolizumab plus bevacizumab in unresectable hepatocellular carcinoma. N Engl J Med. 2020;382:1894-1905.

24. Terashima $T$, Yamashita $T$, Sunagozawa $\mathrm{H}$, et al. Analysis of the liver functional reserve of patients with advanced hepatocellular carcinoma undergoing sorafenib treatment: prospects for regorafenib therapy. Hepatol Res. 2018;48:956-966.

25. Hiraoka A, Kumada T, Atsukawa M, et al. Early relative change in hepatic function with Lenvatinib for Unresectable hepatocellular carcinoma. Oncology. 2019;97:334-340.

26. Sangro B, Park J, Finn R, et al. CheckMate 459: long-term (minimum follow-up 33.6 months) servival outcomes with nivolmab versus sorafenib as first-line treatment in patients with advanced hepatocellular carcinoma. Ann Oncol. 2020;31:241-242.

27. Kudo M, Galle PR, Brandi G, et al. Effect of ramucirumab on ALBI grade in patients with advanced HCC: results from REACH and REACH-2. JHEP Rep. 2021;3:100215.

28. Kuzuya T, Asahina Y, Tsuchiya $\mathrm{K}$, et al. Early decrease in alpha-fetoprotein, but not des-gammacarboxy prothrombin, predicts sorafenib efficacy in patients with advanced hepatocellular carcinoma. Oncology. 2011;81:251-258.

29. Kuzuya T, Ishigami M, Ishizu Y, et al. Fever within 2 weeks of Sorafenib therapy predicts favorable treatment efficacy in patients with advanced hepatocellular carcinoma. Oncology. 2016;91:261-266.

30. Kodama K, Kawaoka T, Namba M, et al. Correlation between early tumor marker response and imaging response in patients with advanced hepatocellular carcinoma treated with lenvatinib. Oncology. 2019;97:75-81.

31. Lee PC, Chao Y, Chen MH, Lan KH, et al. Predictors of response and survival in immune checkpoint inhibitor-treated unresectable hepatocellular carcinoma. Cancers. 2020;12:182.

32. Aoki T, Kudo M, Ueshima $\mathrm{K}$, et al. Exploratory analysis of lenvatinib therapy in patients with unresectable hepatocellular carcinoma who have failed prior PD-1/PD-L1 checkpoint blockade. 
Figures

A. Overall survival $(n=52)$

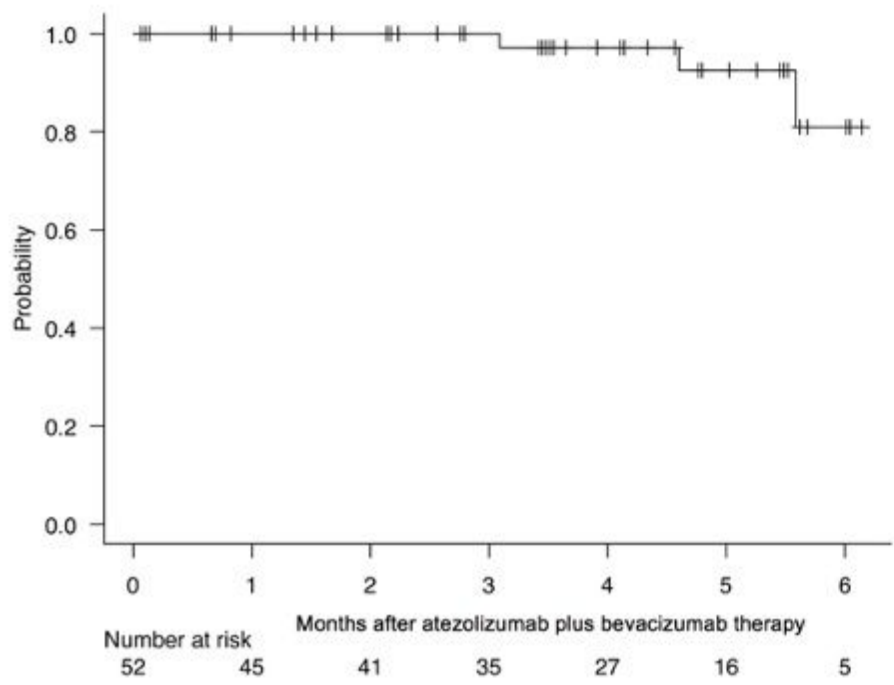

B. Progression-free survival $(\mathrm{n}=38)$

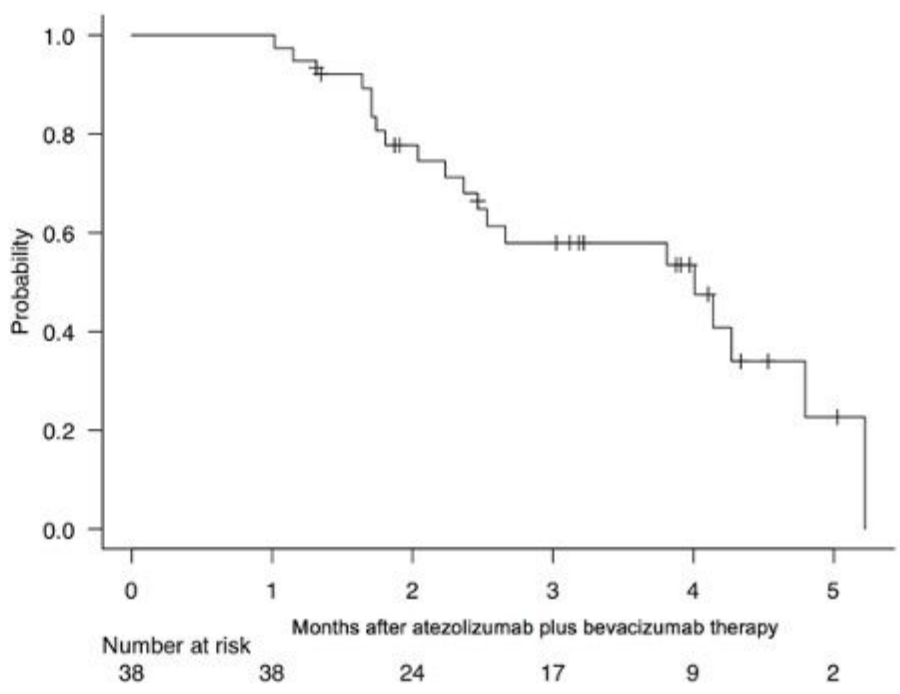

Figure 1

Overall survival (A) and progression-free survival (B) in all patients 

A. Overall survival $(n=23)$
B. Progression-free survival $(n=17)$
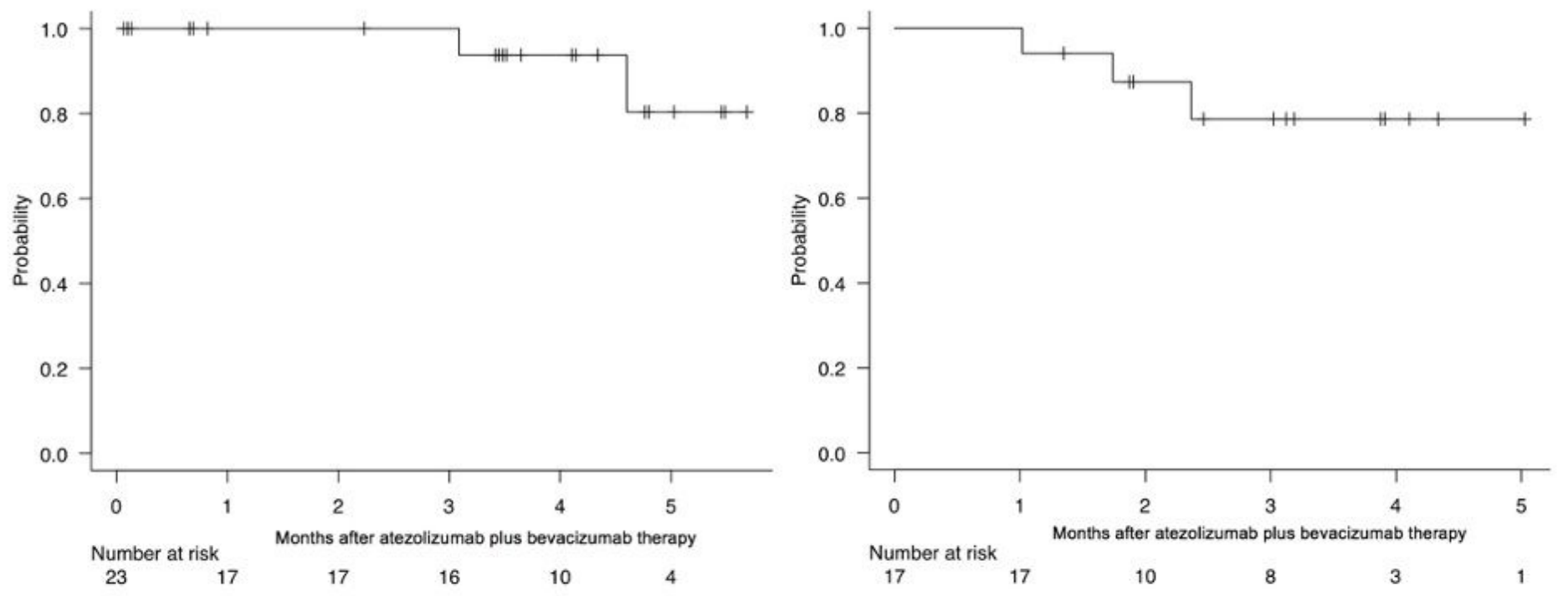

\section{Figure 2}

Overall survival (A) and progression-free survival (B) in patients receiving atezolizumab plus bevacizumab as 1 st-line therapy 


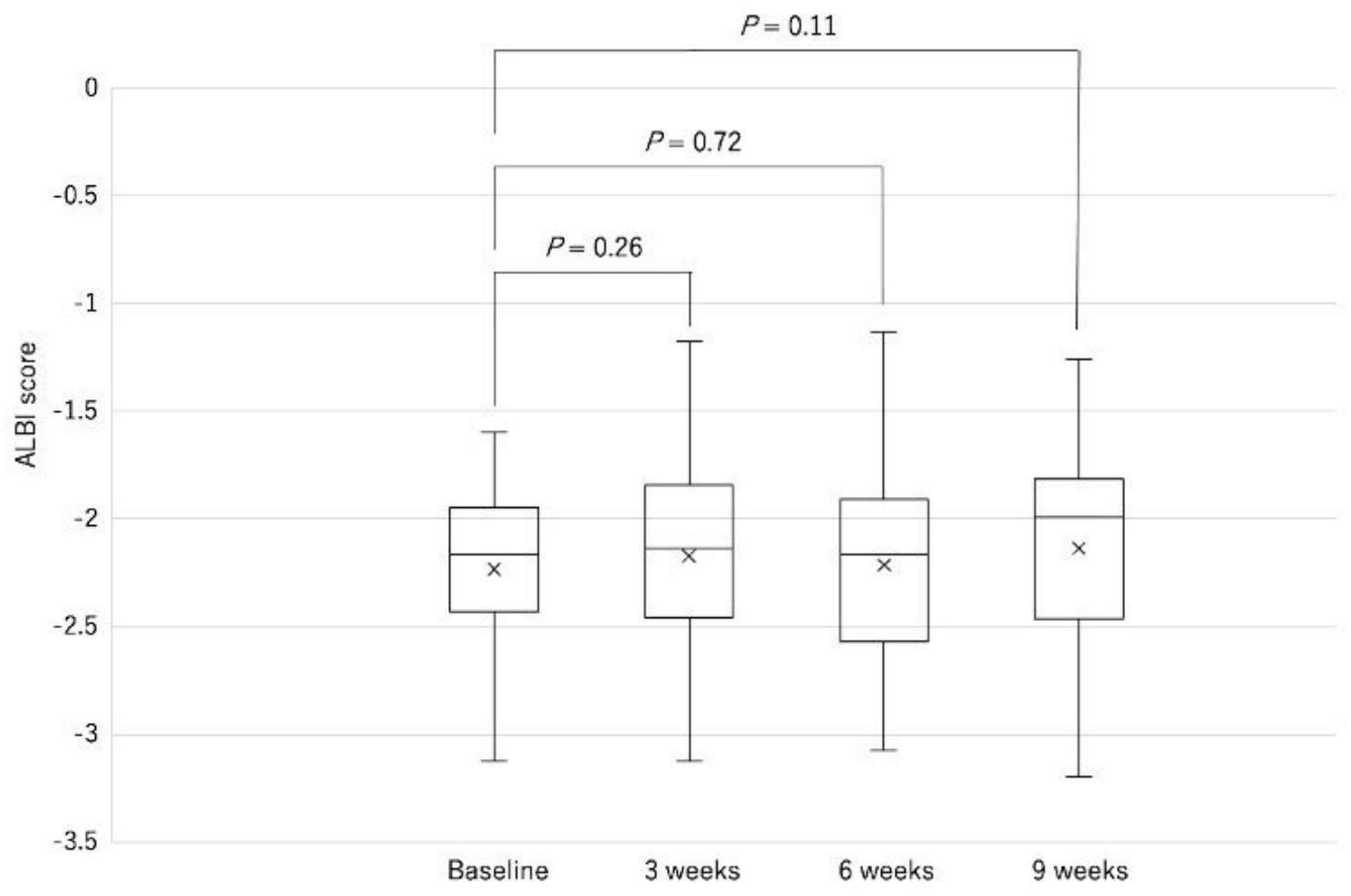

Figure 3

Changes in the median ALBI score $(n=28)$ ALBI score, albumin-bilirubin score 
A. First-line treatment $(n=14)$

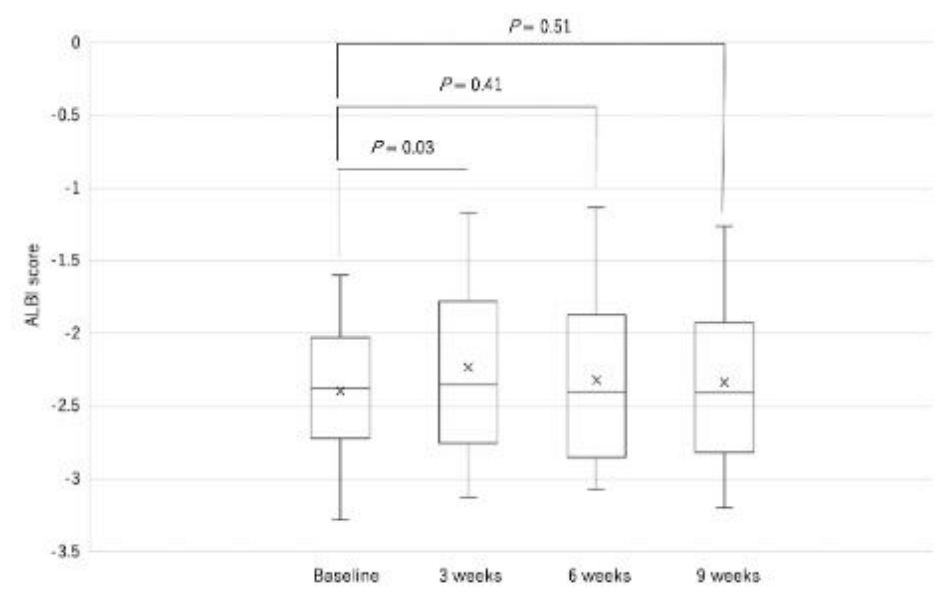

B. Second- or later-line treatment $(n=14)$

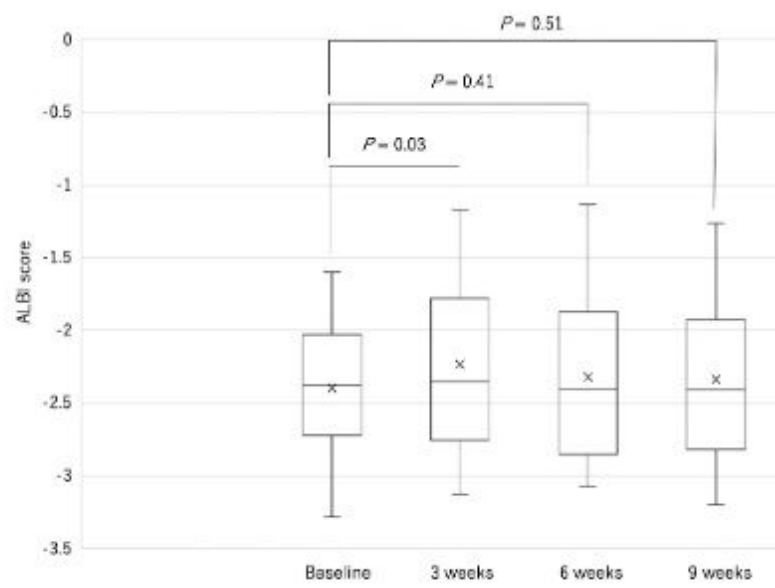

\section{Figure 4}

Changes the in median ALBI score among patients receiving atezolizumab plus bevacizumab as 1 st-line treatment $(n=14)(A)$ and those receiving it as 2 nd- or later-line treatment $(n=14)(B)$ 


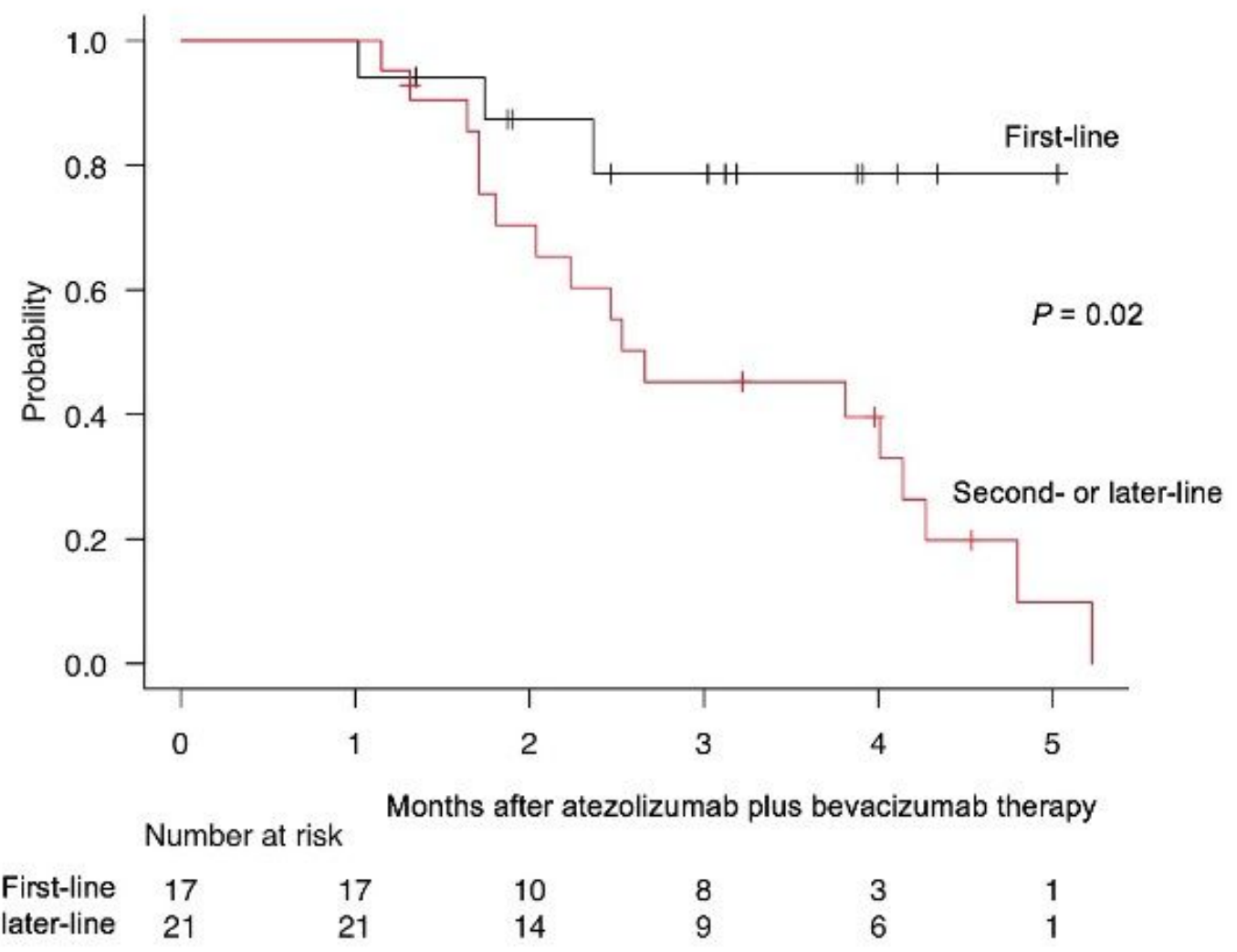

Figure 5

Time to progression in the 1st-line group and the 2nd- or later-line group First-line group: received atezolizumab plus bevacizumab as 1st-line therapy Second- or later-line group: received atezolizumab plus bevacizumab as 2nd-, 3rd-, 4th-, 5th-, or 6th-line therapy 
A. At 3 weeks of treatment

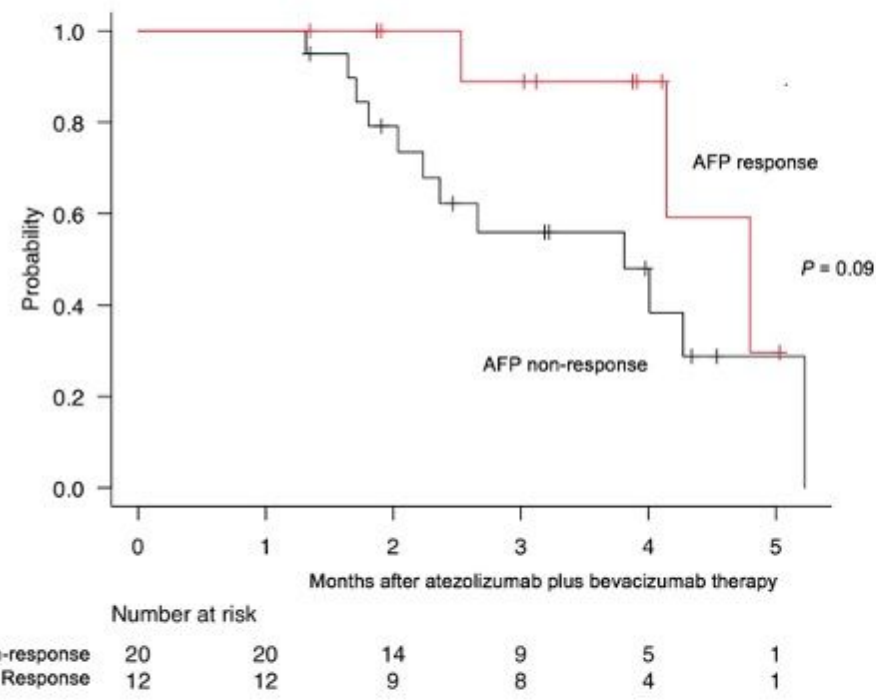

B. At 6 weeks of treatment

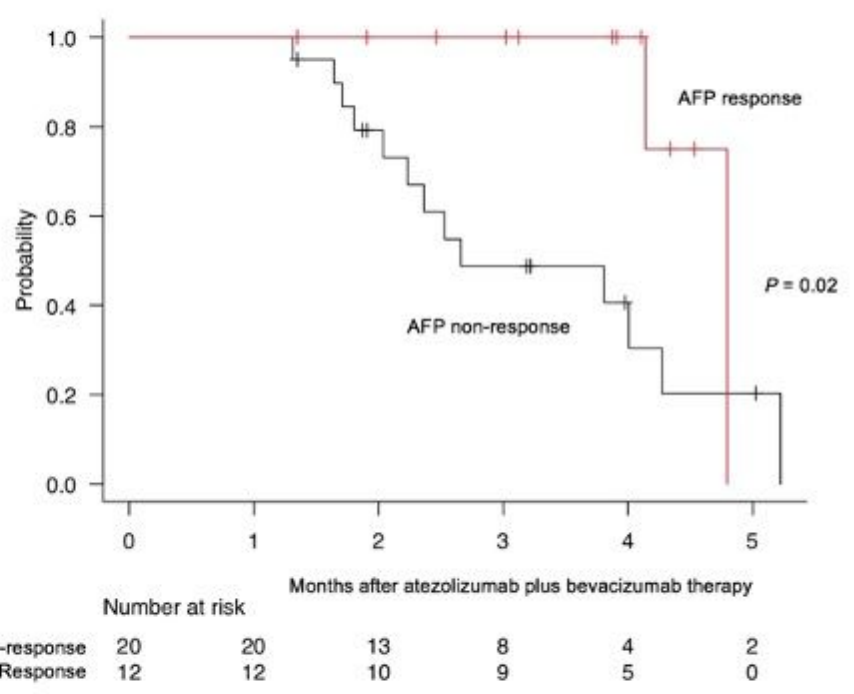

\section{Figure 6}

The comparison of time to progression between patients with and without an AFP response (reduction $\geq 20 \%$ from baseline) at 3 weeks (A) and 6 weeks (B) Abbreviations: AFP, alpha-fetoprotein. 
A. At 3 weeks of treatment

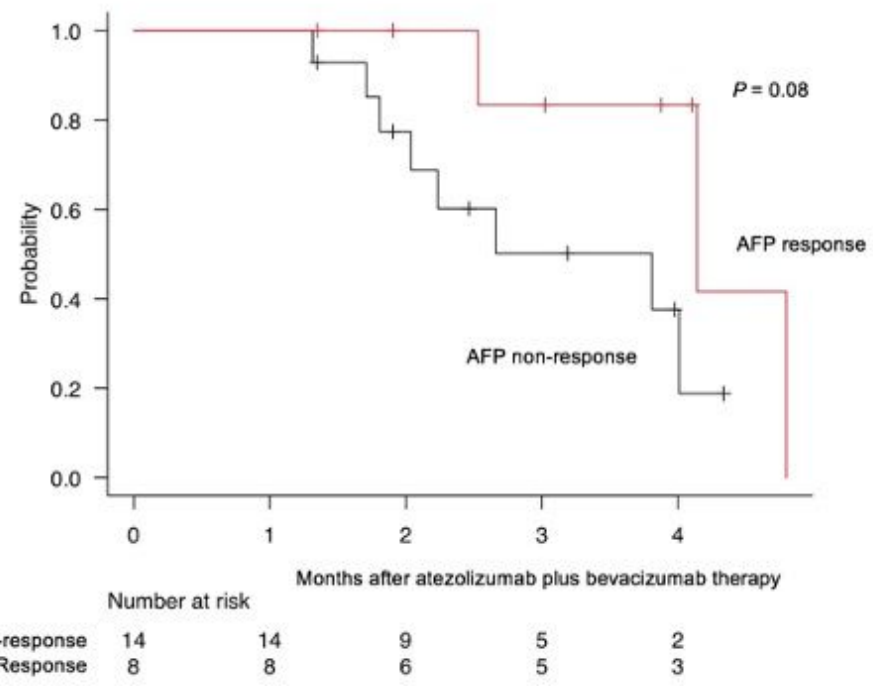

B. At 6 weeks of treatment

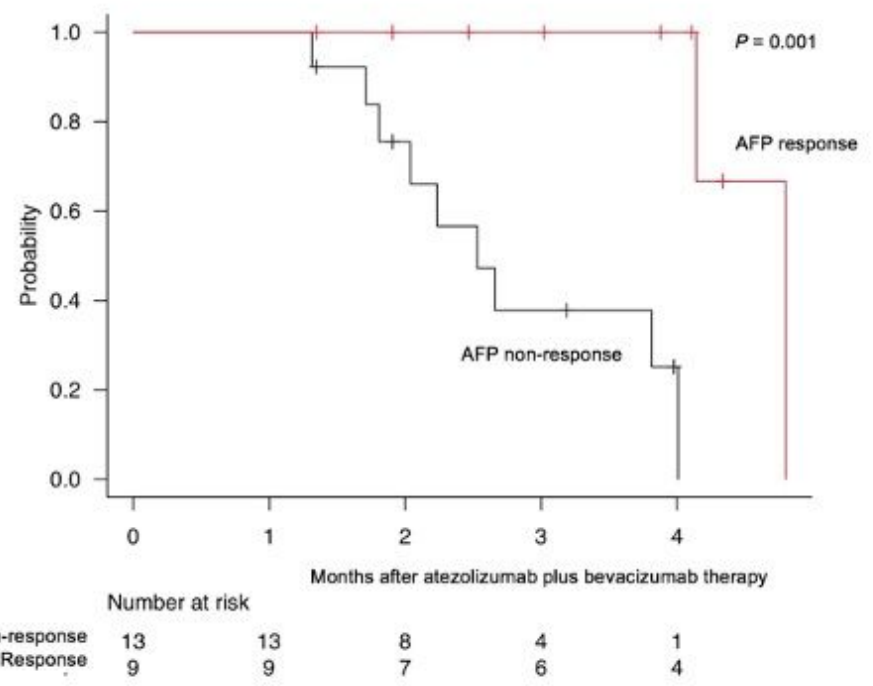

\section{Figure 7}

The comparison of time to progression between patients with and without an AFP response (reduction $>20 \%$ from baseline and baseline AFP $\geq 20 \mathrm{ng} / \mathrm{mL}$ ) at 3 weeks (A) and 6 weeks (B) Abbreviations: AFP, alpha-fetoprotein. 


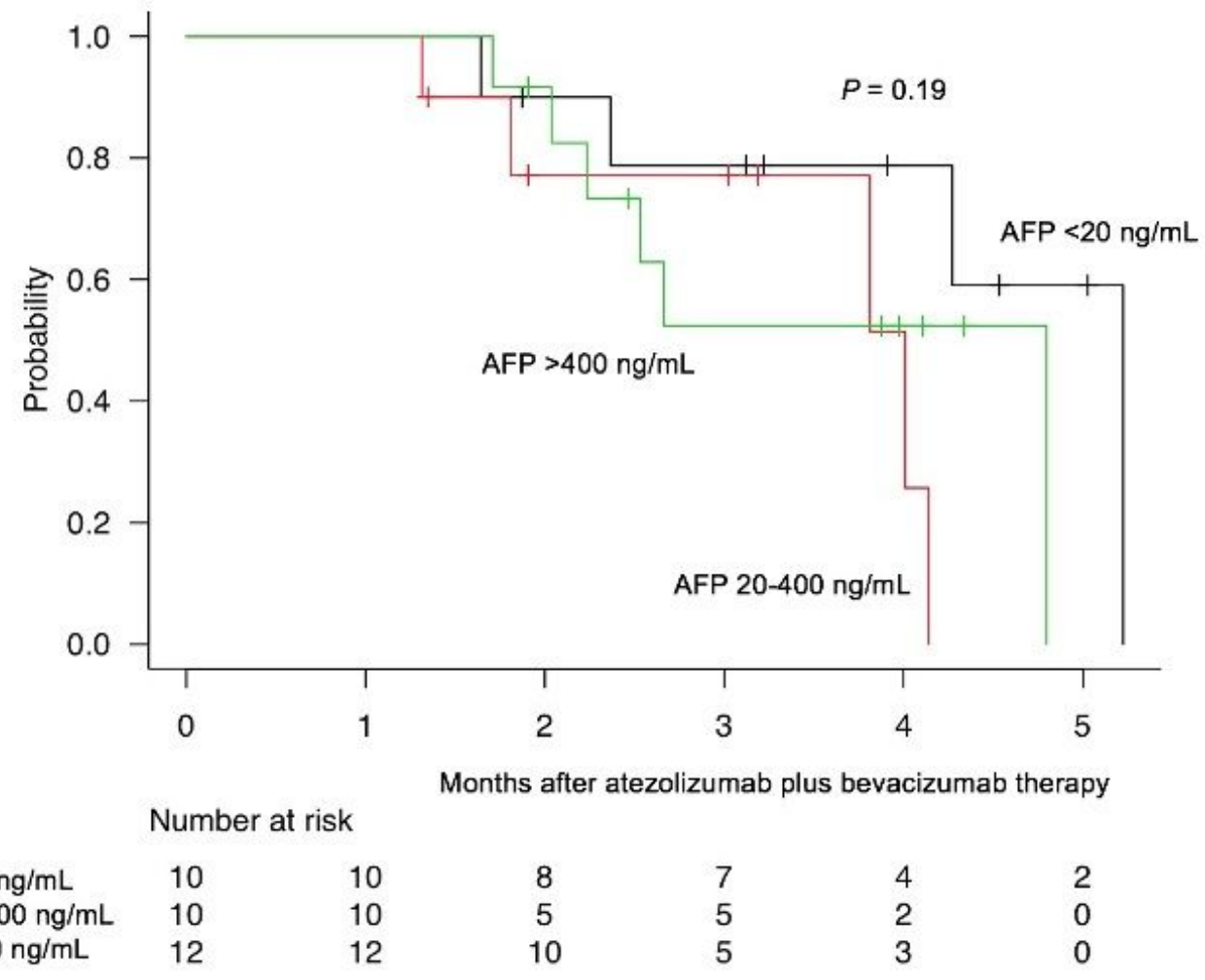

Figure 8

The comparison of time to progression among baseline AFP in patients with an AFP response at 6 weeks Abbreviation: AFP, alpha-fetoprotein. 